\title{
ETHzürich
}

ETH Library

\section{Ein Tropfenakkreszenzmodell in Atmosphäre von homogen isotroper Turbulenz}

\section{Doctoral Thesis}

Author(s):

Gyr, Albert

Publication date:

1965

Permanent link:

https://doi.org/10.3929/ethz-a-000087867

Rights / license:

In Copyright - Non-Commercial Use Permitted 
Prom. Nr. 3599

\title{
Ein Tropfenakkreszenzmodell in Atmosphäre von homogen isotroper Turbulenz
}

\author{
VON DER \\ EIDGENÖSSISCHEN TECHNISCHEN HOCHSCHULE \\ IN ZƯRICH \\ ZUR ERLANGUNG \\ DER WURDE EINES DOKTORS DER NATURWISSENSCHAFTEN \\ GENEHNIGTE \\ PROMOTIONSARBEIT \\ VORGELEGT VON \\ ALBERT GYR \\ dipl. phys. ETH \\ VON \\ USTER und ZURICH
}

Referent: Herr Prof. Dr. M. Fierz

Korreferent: Herr Dr. M. de Quervain 
Tabelle I

\begin{tabular}{|c|c|c|c|c|c|c|c|}
\hline$a$ & $\dot{\varphi}$ & $\tau_{a}$ & $R_{i}$ & $R_{p}$ & $g(a)$ & $N\left(\dot{p}^{2}\right)$ & {$[c \cdot g s]$} \\
\hline \multirow[t]{4}{*}{12,58} & 0,205 & \multirow[t]{4}{*}{3,570} & 12,59 & 32,45 & \multirow[t]{4}{*}{0,141} & 5,800 & $0,458 \cdot 10^{-6}$ \\
\hline & 0,289 & & 12,59 & 45,75 & & 9,550 & 1,453 \\
\hline & 0,354 & & 12,60 & 56,00 & & 5,800 & 1,186 \\
\hline & 0,409 & & 12,60 & 64,80 & & 1,295 & 0,354 \\
\hline \multirow[t]{4}{*}{6,29} & 0,314 & \multirow[t]{4}{*}{2,250} & 6,29 & 12,40 & \multirow[t]{4}{*}{0,113} & 2,460 & 0,042 \\
\hline & 0,444 & & 6,29 & 17,50 & & 4,050 & 0,149 \\
\hline & 0,544 & & 6,29 & 21,45 & & 2,460 & 0,133 \\
\hline & 0,628 & & 6,30 & 24,80 & & 0,549 & 0,081 \\
\hline \multirow[t]{4}{*}{4,19} & 0,316 & \multirow[t]{4}{*}{1,715} & 4,19 & 5,54 & \multirow[t]{4}{*}{0,097} & 2,420 & 0,007 \\
\hline & 0,447 & & & 7,84 & & 3,990 & 0,035 \\
\hline & 0,547 & & & 9,60 & & 2,420 & 0,035 \\
\hline & 0,632 & & & 11,10 & & 0,540 & 0,011 \\
\hline \multirow[t]{4}{*}{3,14} & 0,389 & \multirow[t]{4}{*}{1,416} & 3,14 & 3,84 & \multirow[t]{4}{*}{0,083} & 1,595 & 0 \\
\hline & 0,545 & & & 5,36 & & 2,630 & 0,011 \\
\hline & 0,675 & & & 6,65 & & 1,595 & 0,004 \\
\hline & 0,779 & & & 7,68 & & 0,356 & 0,004 \\
\hline \multirow[t]{4}{*}{2,51} & 0,408 & \multirow[t]{4}{*}{1,220} & 2,51 & kein* & \multirow[t]{4}{*}{0,053} & 1,455 & - \\
\hline & 0,577 & & & 3,63 & & 2,400 & 0,002 \\
\hline & 0,706 & & & 4,45 & & 1,455 & 0,006 \\
\hline & 0,816 & & & 5,14 & & 0,325 & 0 \\
\hline \multirow[t]{4}{*}{2,09} & 0,424 & \multirow[t]{4}{*}{1,079} & 2,09 & kein* & \multirow[t]{4}{*}{0,031} & 1,345 & - \\
\hline & 0,599 & & & 2,65 & & 2,220 & 0,001 \\
\hline & 0,734 & & & 3,20 & & 1,345 & 0 \\
\hline & 0,847 & & & 3,69 & & 0,301 & 0 \\
\hline \multirow[t]{4}{*}{1,79} & 0,405 & \multirow[t]{4}{*}{0,973} & 1,79 & kein* & \multirow[t]{4}{*}{0,013} & 1,475 & - \\
\hline & 0,573 & & & 1,84 & & 2,430 & 0 \\
\hline & 0,701 & & & 2,25 & & 1,475 & 0 \\
\hline & 0,810 & & & 2,60 & & 0,329 & 0 \\
\hline \multirow[t]{4}{*}{1,57} & 0,398 & \multirow[t]{4}{*}{0,893} & 1,57 & kein* & \multirow[t]{4}{*}{0,006} & 1,525 & - \\
\hline & 0,563 & & & kein* & & 2,510 & - \\
\hline & 0,689 & & & 1,70 & & 1,525 & 0 \\
\hline & 0,796 & & & 1,97 & & 0,340 & 0 \\
\hline
\end{tabular}

$\mathrm{Z}=3,972 \cdot 10^{-6}$

* $v\left(t_{0}, a\right)<1$, diese Wirbel können keine Vereinigungszone besitzen.

Der Vorteil, die Messung der Turbulenzverhältnisse eines Windkanalversuches zur Berechnung eines Beispieles zu verwenden, liegt in der Reproduzierbarkeit der Verhältnisse, und auch in der Möglichkeit, das hier eingeführte Tropfenakkreszenz-Modell experimentell nachzuweisen.

Tatsächlich stellt man in Krümmern von Ventilationsanlagen, also an Orten, die ein den Windkanalversuchen sehr ähnliches Turbulenzfeld haben werden, das Ausscheiden von Tropfen fest $^{9}$ ).

Diese Tropfenbildung in einem Windkanal experimentell zu erfassen, wäre schon deshalb reizvoll, weil uns dies die Möglichkeit gäbe, vom Tropfenspektrum einer Wolke ausgehend, Rückschlüsse auf die Turbulenzverhältnisse in derselben zu ziehen.

\section{Zusammenfassung}

In dieser Arbeit wird gezeigt, dass ein stationär, isotrop homogenes Turbulenzfeld aus einzelnen Wirbelballen aufgebaut werden kann, deren Wirbelzentren sich wie

9) Persönliche Mitteilung von Herrn WiLd, Techn. Leiter der Stäfa Ventilator AG. 
starre Körper verhalten und die durch Potentialwirbel gegenüber der Umgebung abgegrenzt sind. Diese Wirbel haben die Eigenschaft, während ihrer Zerfallsperiode (Lebensdauer) in einem Teilgebiet des Potentialwirbels auf Grund eines strömungsmechanischen Vorganges einzelne Tropfen zur Vereinigung miteinander zu bringen. Dieser Akkreszenzprozess ist dabei unabhängig von der ursprünglichen Grössenverteilung der Tropfen und bezieht sich hauptsächlich auf Tropfen zwischen 10-50 $\mu$ Durchmesser.

In einem Beispiel wird die Akkreszenzdichte für ein experimentell ermitteltes Turbulenzfeld bestimmt.

\section{Appendix I}

Die Annahme, dass die Quadrate der Winkelgeschwindigkeit des starren Kernes für ein festes $k$ normal verteilt sind, erfolgt auf Grund von Turbulenzuntersuchungen im Wasser, wie sie von H. A. EINSTEIN und E. A. EL-SAMNI [17] durchgeführt wurden. Die beiden Autoren fanden, dass für eine Wirbelsorte, die durch die Grösse der eingebauten Rauhheit (Halbkugeln) in ihrem häufigsten Durchmesser bestimmt ist, normal verteilte Schubkräfte auftreten. Diese Schubkräfte sind aber proportional den Quadraten der Geschwindigkeiten, also

$$
K_{\tau} \sim v^{2}\left(k_{j}\right) \sim \dot{\varphi}_{j}^{2}
$$

Die experimentellen Untersuchungen zeigen aber auch, dass die Gaussverteilung, Gleichung (29), wie sie nach den obigen Überlegungen angesetzt wurde, ihre Wendetangente bei $0,5 c_{j}^{2}$ besitzt, was das noch zu bestimmende $h$ festlegt

$$
h==\frac{\sqrt{2}}{c_{j}^{2}} .
$$

Die Frage, die sich hier stellen könnte, ist die, ob nicht exaktere Ansätze zur Bestimmung von $N\left(\dot{\varphi}^{2}\right)$ gefunden werden können, zum Beispiel in Form einer analogen Darstellung zur Energieverteilung in Gasen. Wir haben uns bei der statistischen Behandlung in dieser Arbeit davon leiten lassen, dass wir ein gemessenes Fourierspektrum als Ausgangspunkt unserer Berechnung wählten. Dies legte es nahe, ebenfalls von einer experimentellen Untersuchung auszugehen, vor allem, da die vorliegende Darstellung die Rechnung stark vereinfacht. Sollte aber die statistische Betrachtung rein auf der Heisenbergschen Darstellung, Gleichung (25), und damit auf der Berechnung von $v(k)$ aus Gleichung (26) basieren, so ist es angebracht, diese Darstellung neu zu überprüfen.

Mein herzlichster Dank gilt Herrn Professor Dr. M. FIERz und Herrn Dr. M. DE QUervain, die es nach dem schmerzlichen Verlust von Herrn Professor Dr. R. SÄNGer auf sich nahmen, diese Arbeit bis zu ihrem Abschluss zu betreuen.

Ferner bin ich Herrn dipl. Phys. A. GANZ für seine Hilfe bei der Programmierung der numerischen Berechnung zu Dank verpflichtet. 


\section{LITERATURVERZEICHNIS}

[1] H. KÖHLER, Uber die Kondensation an verschieden grossen Kondensationskernen und die Bestimmung ihrer Anzahl, Gerlands Beitrag zur Geophysik 29, 168-186 (1931).

[2] H. KöHLER, The Nucleus and the Growth of Hygroskopic Droplets, Trans. Faraday Soc. $32,1152(1936)$.

[3] W. E. HowelL, The Growth of Cloud Drops in Uniformly Cooled Air, Journ. of Meteorology 6, 134-149 (1949).

[4] I. Bergeron, On the Physics of Clouds and Precipitation, Mém. de l'Union géod. géophys. int. Lisbon (1933).

[5] W. FindeIsen, Die kolloidmeteorologischen Vorgänge der Niederschlagsbildung, Meteo. Z. $55,121-133$ (1938).

[6] I. LANGmuIR and K. B. Blodgetr, A Mathematical Investigation of Water Droplet Trajectories, Army Air Force Techn. Report No. 5418, 66pp, Washington DC (1946).

[7] I. Langmuir and K. B. Blodgetr, The Production of Rain by a Chain Reaction in Cumulus Clouds at Temperature above Freezing, Journ. of Meteorology 5, 175-192 (1948).

[8] W. J. Kотsch, An Example of Colloidal Instability of Clouds in Tropical Latitudes, Bull. Americ. Meteor. Soc. 28, 79-87 (1947).

[9] E. H. Ludlam and B. J. Mason, Growth of a Droplet by Accretion, Handbuch der Physik 48, 497 (1960).

[10] M. Neiburger and C. W. Chien, Computations of the Growth of Cloud Drops by Condensation Using an Electronic Digital Computer, U.C.L.A. Dept. of Meteor., Contribution to Meteor. No. 40.

[11] G. Gotsch und S. Bernasconi, Uber die Kondensation verschiedener Dämpfe bei adiabatischer Expansion, ZAMP 10, 509 (1959).

[12] A. GyR, Theoretical Reflections on the Growth of Droplets under Extreme Cooling Conditions, ZAMP 13, 288-293 (1962).

[13] A. GYR, Die Kleintropfenbewegung in einem homogenen isotropen Turbulenzfeld, ZAMP 14, 449-455 (1963).

[14] C. F. voN WEIzsÄCKer, Das Spehtrum der Turbulenz bei grossen Reynoldschen Zahlen, Z. f. Physik 124, 614-627 (1948).

[15] W. Heisenberg, Zur statistischen Theorie der Turbulenz, Z. f. Physik 124, 628-657 (1948).

[16] R. W. StEWARt and A. A. TownSEnd, Similarity and Self-preservation in Isolropic Turbulence, Phil. Trans-Roy. Soc. London 243A, 359-386 (1951).

[17] H. A. Einstein and E-S. A. El-SAmni, Hydrodynamic Forces on a Rough Wall, Rev. of mod. Phys. 27, 520-524 (1949).

\section{Summary}

In the present work a turbulence model is set up which shows accrescence qualities for small droplets during its time of diffusion dissociation. At the same time the life time of the single element, and by that the quantitative accrescence ratio which originates in an turbulence element, is defined by the general turbulence statistic.

By means of a calculated example it is shown how the accrescence density in one real. situation is being computed for a whole statistical ensemble. The results show good conformity with available experimental data.

(Eingegangen: 14. April 1965.) 